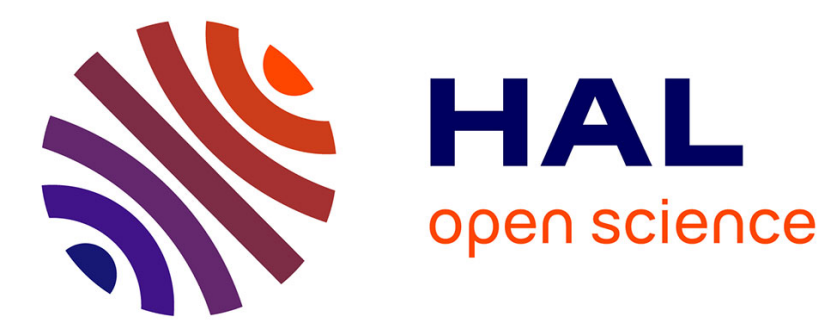

\title{
2D dual-tree complex biorthogonal M-band wavelet transform
}

Caroline Chaux, Jean-Christophe Pesquet, Laurent Duval

\section{To cite this version:}

Caroline Chaux, Jean-Christophe Pesquet, Laurent Duval. 2D dual-tree complex biorthogonal Mband wavelet transform. IEEE International Conference on Acoustics, Speech, and Signal Processing (ICASSP'07), Apr 2007, Honolulu, Hawaii, USA, United States. pp.845-848. hal-00621884

\section{HAL Id: hal-00621884 https://hal.science/hal-00621884}

Submitted on 9 Jul 2013

HAL is a multi-disciplinary open access archive for the deposit and dissemination of scientific research documents, whether they are published or not. The documents may come from teaching and research institutions in France or abroad, or from public or private research centers.
L'archive ouverte pluridisciplinaire HAL, est destinée au dépôt et à la diffusion de documents scientifiques de niveau recherche, publiés ou non, émanant des établissements d'enseignement et de recherche français ou étrangers, des laboratoires publics ou privés. 


\title{
2D DUAL-TREE COMPLEX BIORTHOGONAL $M$-BAND WAVELET TRANSFORM
}

\author{
Caroline Chaux ${ }^{1}$, Jean-Christophe Pesquet ${ }^{1}$ and Laurent Duval ${ }^{2}$
}

\author{
${ }^{1}$ Institut Gaspard Monge and UMR-CNRS 8049 \\ Université de Marne-la-Vallée, Champs-sur-Marne \\ 77454 Marne-la-Vallée, France \\ e-mail: \{chaux,pesquet\}@univ-mlv.fr
}

\author{
${ }^{2}$ Institut Français du Pétrole, IFP \\ Technology, Comp. Sci. and Appl. Math. Div. \\ 92500 Rueil Malmaison, France \\ e-mail : laurent.duval@ifp.fr
}

\begin{abstract}
Dual-tree wavelet transforms have recently gained popularity [1] since they provide low-redundancy directional analyses of images. In our recent work, dyadic real dual-tree decompositions have been extended to the $M$-band case, so adding much flexibility to this analysis tool. In this work, we propose to further extend this framework on two fronts by considering (i) biorthogonal and (ii) complex $M$-band dualtree decompositions. Denoising results are finally provided to demonstrate the validity of the proposed design rules.
\end{abstract}

Index Terms - Wavelet transforms, Hilbert transforms, Image analysis, Image processing, Gaussian noise.

\section{INTRODUCTION}

Natural images often require a transformed domain processing in order to improve their quality (denoising or restoration tasks) or to be efficiently compressed. Powerful tools allowing improved representations of images have been developed in the last ten years. They aim at capturing directional features and often introduce some redundant analysis. They can be classified into different categories: steerable filters, "“lets" transforms including bandelets [2], curvelets [3], contourlets [4], ... Meanwhile, the dual-tree wavelet decomposition has been introduced by N. Kingsbury [5]. This transform is based on a combination of classical wavelet decompositions. It has been further investigated by I. Selesnick [6]. The standard real dual-tree decomposition is 2 times redundant and is nearly shift-invariant. In the 2-band case, in two dimensions, a complex version of this decomposition has been obtained (then, the redundancy becomes equal to 4 ) and, more recently, it has also been extended to biorthogonal representations [7]. The dual-tree transform is interesting for several reasons: good directional analysis, low redundancy, improved shift-invariance property, simplicity of implementation, reduced computational cost,... However, a limiting factor lies in its dyadic structure which introduces some inherent design constraints. For example, it is well-known that is impossible to obtain real, orthogonal, symmetric and compactly supported dyadic wavelets, except for the Haar one. In one of our recent works [8], we have extended the real dual-tree transform to the $M$-band case, which allowed us to gain more freedom in the choice of the filters while providing a more accurate frequency analysis.

In this work, we propose to extend the real 2D dual-tree $M$-band wavelet transform to the biorthogonal complex case, for an arbitrary integer value of the decimation factor $M$. The paper is organized as follow: in Section 2 we show how to design Hilbert pairs of biorthogonal wavelets. Then, Section 3 is devoted to the complex decomposition. Finally, denoising applications are reported in Section 4.

Throughout the paper, the following notations will be used: let $M$ be an integer greater than or equal to 2, $\mathbb{N}_{M}=\{0, \ldots, M-1\}$ and $\mathbb{N}_{M}^{\star}=\{1, \ldots, M-1\}$. Besides, $\widehat{a}$ denotes the Fourier transform of a function $a,\lceil u\rceil$ denotes the upper integer part of a real $u,\left(\delta_{m}\right)_{m \in \mathbb{Z}}$ is the Kronecker sequence (equal to 1 if $m=0$ and 0 otherwise).

\section{BIORTHOGONAL HILBERT PAIRS OF WAVELETS}

We address in this section the problem of constructing a Hilbert pair of $M$-band biorthogonal wavelets in the one dimensional case. A biorthogonal wavelet basis corresponding to a multiresolution analysis of $\mathrm{L}^{2}(\mathbb{R})$ is associated to an analysis and a synthesis filter bank and the problem is then equivalent to the design of $2 M$ filters.

\subsection{Problem statement}

Let us start from an $M$-band biorthogonal wavelet decomposition of $L^{2}(\mathbb{R})$. This decomposition is based on the joint use of two sets of basis functions: $\left(\psi_{m}\right)_{0 \leq m<M}$ and $\left(\widetilde{\psi}_{m}\right)_{0 \leq m<M}$ which satisfy the following scaling equations expressed in the frequency domain:

$$
\begin{aligned}
\forall m \in \mathbb{N}_{M}, \quad \sqrt{M} \widehat{\psi}_{m}(M \omega) & =H_{m}(\omega) \widehat{\psi}_{0}(\omega), \\
\sqrt{M} \widehat{\widetilde{\psi}}_{m}(M \omega) & =\widetilde{H}_{m}(\omega) \widehat{\widetilde{\psi}}_{0}(\omega) .
\end{aligned}
$$

Furthermore, the associated $M$-band filter banks with frequency responses $\left(H_{m}\right)_{0 \leq m<M}$ and $\left(\widetilde{H}_{m}\right)_{0 \leq m<M}$ satisfy per- 
fect reconstruction properties:

$$
\begin{aligned}
& \forall\left(m, m^{\prime}\right) \in \mathbb{N}_{M}^{2}, \\
& \quad \sum_{p=0}^{M-1} \widetilde{H}_{m}\left(\omega+p \frac{2 \pi}{M}\right) H_{m^{\prime}}^{*}\left(\omega+p \frac{2 \pi}{M}\right)=M \delta_{m-m^{\prime}}
\end{aligned}
$$

Similarly to the orthogonal case [8], we want to construct Hilbert pairs of wavelets by defining functions $\left(\psi_{m}^{\mathrm{H}}\right)_{0 \leq m<M}$ and

$\left(\widetilde{\psi_{m}^{\mathrm{H}}}\right)_{0 \leq m<M}$ such that

$$
\begin{array}{ll}
\forall m \in \mathbb{N}_{M}^{\star}, \quad \widehat{\psi}_{m}^{\mathrm{H}}(\omega)=-\imath \operatorname{sign}(\omega) \widehat{\psi}_{m}(\omega) \\
\widehat{\widetilde{\psi}}_{m}^{\mathrm{H}}(\omega)=-\imath \operatorname{sign}(\omega) \widehat{\widetilde{\psi}}_{m}(\omega),
\end{array}
$$

where sign designates the signum function. The filters with frequency responses $\left(G_{m}\right)_{0 \leq m<M}$ and $\left(\widetilde{G}_{m}\right)_{0 \leq m<M}$ associated with these dual basis functions must also satisfy perfect reconstruction conditions:

$$
\begin{aligned}
& \forall\left(m, m^{\prime}\right) \in \mathbb{N}_{M}^{2}, \\
& \quad \sum_{p=0}^{M-1} \widetilde{G}_{m}\left(\omega+p \frac{2 \pi}{M}\right) G_{m^{\prime}}^{*}\left(\omega+p \frac{2 \pi}{M}\right)=M \delta_{m-m^{\prime}}
\end{aligned}
$$

We now study how to design such filters.

\subsection{Dual filter construction}

The Hilbert conditions (3) imply that:

$\forall m \in \mathbb{N}_{M}^{\star}, \quad\left|\widehat{\psi}_{m}^{\mathrm{H}}(\omega)\right|=\left|\widehat{\psi}_{m}(\omega)\right|$ and $\left|\widehat{\widetilde{\psi}}_{m}^{\mathrm{H}}(\omega)\right|=\left|\widehat{\widetilde{\psi}}_{m}(\omega)\right|$.

In addition, by imposing that $\left|\widehat{\psi}_{0}^{\mathrm{H}}(\omega)\right|=\left|\widehat{\psi}_{0}(\omega)\right|$, and $\left|\widehat{\widetilde{\psi}}_{0}^{\mathrm{H}}(\omega)\right|$ $=\left|\widehat{\widetilde{\psi}}_{0}(\omega)\right|$, the scaling equations (1) and their counterparts for the dual wavelets lead to

$$
\begin{aligned}
\forall m \in \mathbb{N}_{M}, \quad G_{m}(\omega)=e^{-\imath \theta_{m}(\omega)} H_{m}(\omega) \\
\widetilde{G}_{m}(\omega)=e^{-\imath \widetilde{\theta}_{m}(\omega)} \widetilde{H}_{m}(\omega),
\end{aligned}
$$

where $\theta_{m}$ and $\widetilde{\theta}_{m}$ are real-valued $2 \pi$-periodic phase functions. Assuming that (2) is satisfied, one can easily check that the perfect reconstruction conditions (4) for the dual filter bank are fulfilled if for all $\left(m, m^{\prime}\right) \in \mathbb{N}_{M}^{2}, \widetilde{\theta}_{m}=\theta_{m}(\bmod 2 \pi)$ and $\theta_{m^{\prime}}-\widetilde{\theta}_{m}=\theta_{m^{\prime}}-\theta_{m}$ is $2 \pi / M$-periodic.

Conditions to be satisfied by $\theta_{m}$ and $\widetilde{\theta}_{m}$ are thus similar to those obtained in the orthogonal case [8]. It can be deduced that

$$
\begin{array}{r}
\forall p \in\left\{0, \ldots,\left\lceil\frac{M}{2}\right\rceil-1\right\}, \forall \omega \in\left[p \frac{2 \pi}{M},(p+1) \frac{2 \pi}{M}[,\right. \\
\tilde{\theta}_{0}(\omega)=\theta_{0}(\omega)=\left(d+\frac{1}{2}\right)(M-1) \omega-p \pi,
\end{array}
$$

and

$$
\begin{gathered}
\forall m \in\{1, \ldots, M-1\}, \\
\tilde{\theta}_{m}(\omega)=\theta_{m}(\omega)= \begin{cases}\frac{\pi}{2}-\left(d+\frac{1}{2}\right) \omega & \text { if } \omega \in] 0,2 \pi[, \\
0 & \text { if } \omega=0,\end{cases}
\end{gathered}
$$

where $d \in \mathbb{Z}$.

\section{COMPLEX 2D DUAL-TREE WAVELET TRANSFORM}

Our objective in this section is to extend the complex dual tree transform to the $M$-band case. We will see that an increased number of directions can be selected and show how to implement the associated decomposition. Finally, the problem of finding an optimized reconstruction will be discussed.

\subsection{Direction selection}

For all $m \in \mathbb{N}_{M}^{\star}$ and $\epsilon \in\{-1,1\}$, let us define the following complex-valued functions from the wavelets $\left(\psi_{m}\right)_{m \in \mathbb{N}_{M}^{\star}}$ used for the analysis stage:

$$
\forall x \in \mathbb{R}, \quad \psi_{m}^{\epsilon}(x)=\psi_{m}(x)+\imath \epsilon \psi_{m}^{\mathrm{H}}(x) .
$$

If $\epsilon=1$ (resp. $\epsilon=-1$ ), this corresponds to an analytic (resp. anti-analytic) wavelet. By considering all the possible combinations of tensor products of such wavelets, we obtain: for all $\left(m_{1}, m_{2}\right) \in \mathbb{N}_{M}^{\star 2},\left(\epsilon_{1}, \epsilon_{2}\right) \in\{-1,1\}^{2}$ and $\left(x_{1}, x_{2}\right) \in$ $\mathbb{R}^{2}$,

$$
\begin{aligned}
& \psi_{m_{1}}^{\epsilon_{1}}\left(x_{1}\right) \psi_{m_{2}}^{\epsilon_{2}}\left(x_{2}\right)= \\
& \quad \psi_{m_{1}}\left(x_{1}\right) \psi_{m_{2}}\left(x_{2}\right)-\epsilon_{1} \epsilon_{2} \psi_{m_{1}}^{\mathrm{H}}\left(x_{1}\right) \psi_{m_{2}}^{\mathrm{H}}\left(x_{2}\right) \\
& \quad+\imath\left(\epsilon_{1} \psi_{m_{1}}^{\mathrm{H}}\left(x_{1}\right) \psi_{m_{2}}\left(x_{2}\right)+\epsilon_{2} \psi_{m_{1}}\left(x_{1}\right) \psi_{m_{2}}^{\mathrm{H}}\left(x_{2}\right)\right)
\end{aligned}
$$

which reads in the Fourier domain:

$$
\begin{aligned}
& \widehat{\psi}_{m_{1}}^{\epsilon_{1}}\left(\omega_{1}\right) \widehat{\psi}_{m_{2}}^{\epsilon_{2}}\left(\omega_{2}\right)= \\
& \quad\left(1+\epsilon_{1} \operatorname{sign}\left(\omega_{1}\right)\right)\left(1+\epsilon_{2} \operatorname{sign}\left(\omega_{2}\right)\right) \widehat{\psi}_{m_{1}}\left(\omega_{1}\right) \widehat{\psi}_{m_{2}}\left(\omega_{2}\right)
\end{aligned}
$$

The latter expression shows that, depending on the choice of $\epsilon_{1}$ and $\epsilon_{2}$, it is possible to select each quadrant of the frequency plane as represented in Fig. 1.

Let us consider the real part (RP) and the imaginary part (IP) of the expression in (6) and consider once again all possible situations. This leads to the two following cases:

$$
\begin{aligned}
& >\epsilon_{1}=\epsilon_{2}=1 \text { or } \epsilon_{1}=\epsilon_{2}=-1: \\
& \text { RP: } \psi_{m_{1}}\left(x_{1}\right) \psi_{m_{2}}\left(x_{2}\right)-\psi_{m_{1}}^{\mathrm{H}}\left(x_{1}\right) \psi_{m_{2}}^{\mathrm{H}}\left(x_{2}\right) \\
& \text { IP: } \operatorname{sign}\left(\epsilon_{1}\right)\left(\psi_{m_{1}}^{\mathrm{H}}\left(x_{1}\right) \psi_{m_{2}}\left(x_{2}\right)+\psi_{m_{2}}^{\mathrm{H}}\left(x_{2}\right) \psi_{m_{1}}\left(x_{1}\right)\right)
\end{aligned}
$$




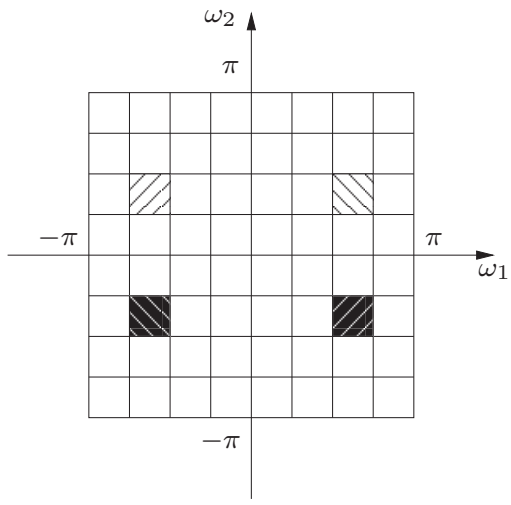

Fig. 1. Direction selection in the $2 \mathrm{D}$ frequency plane for $M=$ $4, j=1$ and $\left(m_{1}, m_{2}\right)=(2,1)$. The four crosshatched areas are separated using tensor products of analytic/anti-analytic wavelets.

$$
\begin{aligned}
> & \epsilon_{1}=-\epsilon_{2}=1 \text { or } \epsilon_{1}=-\epsilon_{2}=-1 \text { : } \\
& \text { RP: } \psi_{m_{1}}\left(x_{1}\right) \psi_{m_{2}}\left(x_{2}\right)+\psi_{m_{1}}^{\mathrm{H}}\left(x_{2}\right) \psi_{m_{2}}^{\mathrm{H}}\left(x_{2}\right) \\
& \text { IP: } \operatorname{sign}\left(\epsilon_{1}\right)\left(\psi_{m_{1}}^{\mathrm{H}}\left(x_{1}\right) \psi_{m_{2}}\left(x_{2}\right)-\psi_{m_{2}}^{\mathrm{H}}\left(x_{2}\right) \psi_{m_{1}}\left(x_{1}\right)\right) .
\end{aligned}
$$

In other words, in order to implement the decomposition onto $\left(\psi_{m_{1}}^{\epsilon_{1}}\left(x_{1}\right) \psi_{m_{2}}^{\epsilon_{2}}\left(x_{2}\right)\right)_{\left(m_{1}, m_{2}\right) \in \mathbb{N}_{M}^{\star 2}}$, we need four separable 2D wavelet analyses (see the corresponding filter bank structures in Fig. 2). The two upper analyses, which are also present in the real parts, are implemented in the real biorthogonal case.

These four $M$-band wavelet decompositions generate coefficients denoted by $c_{j, \mathbf{m}}^{\bullet \bullet}[\mathbf{k}], c_{j, \mathbf{m}}^{\mathrm{HH}}[\mathbf{k}], c_{j, \mathbf{m}}^{\mathrm{H} \bullet}[\mathbf{k}]$ and $c_{j, \mathbf{m}}^{\bullet \mathrm{H}}[\mathbf{k}]$ where $j \in \mathbb{Z}$ denotes the resolution level, $\mathbf{m}=$ $\left(m_{1}, m_{2}\right) \in \mathbb{N}_{M}^{2}$ is the frequency band index and $\mathbf{k} \in \mathbb{Z}^{2}$ is the spatial position. Finally, to obtain the desired directional analysis, we compute the inner product of the image to be processed with the RP and IP terms of the two-dimensional wavelets as previously expressed. This is equivalent to perform the following linear combinations of the subbands: for all $\mathbf{m} \in \mathbb{N}_{M}^{\star 2}$,

$$
\begin{aligned}
& d_{j, \mathbf{m}}^{\bullet \bullet}[\mathbf{k}]=\frac{1}{\sqrt{2}}\left(c_{j, \mathbf{m}}^{\bullet \bullet}[\mathbf{k}]+c_{j, \mathbf{m}}^{\mathrm{HH}}[\mathbf{k}]\right) \\
& d_{j, \mathbf{m}}^{\mathrm{HH}}[\mathbf{k}]=\frac{1}{\sqrt{2}}\left(c_{j, \mathbf{m}}^{\bullet \bullet}[\mathbf{k}]-c_{j, \mathbf{m}}^{\mathrm{HH}}[\mathbf{k}]\right) \\
& d_{j, \mathbf{m}}^{\mathrm{H} \bullet}[\mathbf{k}]=\frac{1}{\sqrt{2}}\left(c_{j, \mathbf{m}}^{\mathrm{H} \bullet}[\mathbf{k}]+c_{j, \mathbf{m}}^{\bullet \mathrm{H}}[\mathbf{k}]\right) \\
& d_{j, \mathbf{m}}^{\bullet \mathrm{H}}[\mathbf{k}]=\frac{1}{\sqrt{2}}\left(c_{j, \mathbf{m}}^{\mathrm{H} \bullet}[\mathbf{k}]-c_{j, \mathbf{m}}^{\bullet \mathrm{H}}[\mathbf{k}]\right) .
\end{aligned}
$$

\subsection{Prefiltering stage}

In a digital implementation of the dual-tree decomposition, prefilters must be added to make the transition from the ana$\log$ formalism to discrete processing [8].

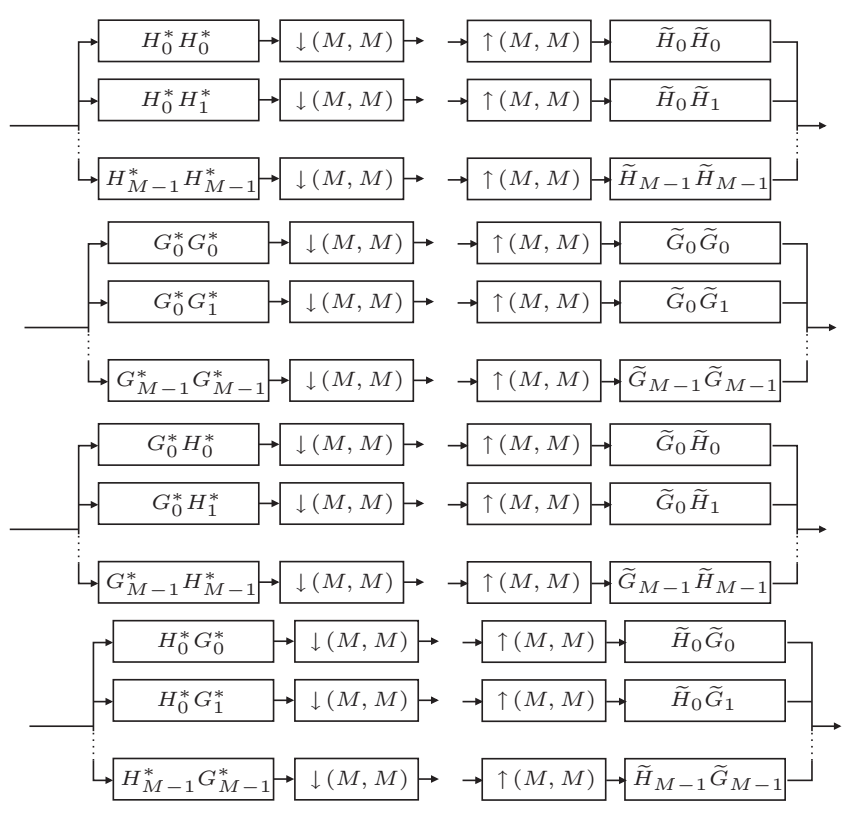

Fig. 2. Two-dimensional analysis/synthesis filter banks corresponding to a biorthogonal complex wavelet transform.

Assume that the digital image $\left(r_{k_{1}, k_{2}}\right)_{\left(k_{1}, k_{2}\right)}$ to be analyzed is related to its analog version $r\left(x_{1}, x_{2}\right)$ by the relation:

$$
r\left(x_{1}, x_{2}\right)=\sum_{\left(k_{1}, k_{2}\right)} r_{k_{1}, k_{2}} \chi\left(x_{1}-k_{1}, x_{2}-k_{2}\right)
$$

where $\chi$ is an interpolation function in $\mathrm{L}^{2}\left(\mathbb{R}^{2}\right)$. To perform the first separable wavelet decomposition, we need to determine the approximation coefficients: $c_{0,(0,0)}\left[k_{1}, k_{2}\right]=$ $\left\langle r\left(x_{1}, x_{2}\right), \psi_{0}\left(x_{1}-k_{1}\right) \psi_{0}\left(x_{2}-k_{2}\right)\right\rangle$. Similar approximations have to be computed for the three remaining trees. It is easy to show that these operations can be performed by applying four digital prefilters with frequency responses:

$$
\begin{aligned}
& F_{1}\left(\omega_{1}, \omega_{2}\right)=\sum_{p_{1}=-\infty}^{\infty} \sum_{p_{2}=-\infty}^{\infty} \widehat{\chi}\left(\omega_{1}+2 p_{1} \pi, \omega_{2}+2 p_{2} \pi\right) \\
& \widehat{\psi}_{0}^{*}\left(\omega_{1}+2 p_{1} \pi\right) \widehat{\psi}_{0}^{*}\left(\omega_{2}+2 p_{2} \pi\right) \\
& F_{2}\left(\omega_{1}, \omega_{2}\right)=\sum_{p_{1}=-\infty}^{\infty} \sum_{p_{2}=-\infty}^{\infty} \widehat{\chi}\left(\omega_{1}+2 p_{1} \pi, \omega_{2}+2 p_{2} \pi\right) \\
&\left(\widehat{\psi}_{0}^{\mathrm{H}}\left(\omega_{1}+2 p_{1} \pi\right)\right)^{*}\left(\widehat{\psi}_{0}^{\mathrm{H}}\left(\omega_{2}+2 p_{2} \pi\right)\right)^{*} \\
& F_{3}\left(\omega_{1}, \omega_{2}\right)=\sum_{p_{1}=-\infty}^{\infty} \sum_{p_{2}=-\infty}^{\infty} \widehat{\chi}\left(\omega_{1}+2 p_{1} \pi, \omega_{2}+2 p_{2} \pi\right) \\
&\left(\widehat{\psi}_{0}^{\mathrm{H}}\left(\omega_{1}+2 p_{1} \pi\right)\right)^{*} \widehat{\psi}_{0}^{*}\left(\omega_{2}+2 p_{2} \pi\right) \\
& F_{4}\left(\omega_{1}, \omega_{2}\right)=\sum_{p_{1}=-\infty}^{\infty} \sum_{p_{2}=-\infty}^{\infty} \widehat{\chi}\left(\omega_{1}+2 p_{1} \pi, \omega_{2}+2 p_{2} \pi\right) \\
& \widehat{\psi}_{0}^{*}\left(\omega_{1}+2 p_{1} \pi\right)\left(\widehat{\psi}_{0}^{\mathrm{H}}\left(\omega_{2}+2 p_{2} \pi\right)\right)^{*} .
\end{aligned}
$$




\subsection{Reconstruction}

As already mentioned, the complex dual-tree transform has a redundancy of a factor 4 . As a consequence, the reconstruction is not unique and we have to pay attention to the choice of the synthesis scheme.

Let $\mathbf{r} \in \ell^{2}\left(\mathbb{Z}^{2}\right)$ be the vector of image values and $\mathbf{c}^{\bullet \bullet}, \mathbf{c}^{\mathrm{HH}}$, $\mathbf{c}^{\mathrm{H} \bullet}, \mathbf{c}^{\bullet \mathrm{H}}$ denote the coefficient vectors generated by the complex dual-tree transform. The linear combination of the subbands is not taken into account as it reduces to a basic isometry. The global decomposition operator can be written as

$$
\mathbf{D}_{\mathbf{c}}: \mathbf{r} \mapsto\left(\begin{array}{c}
\mathbf{c}^{\bullet \bullet} \\
\mathbf{c}^{\mathrm{HH}} \\
\mathbf{c}^{\mathrm{H} \bullet} \\
\mathbf{c}^{\bullet \mathrm{H}}
\end{array}\right)=\left(\begin{array}{l}
\mathbf{D}_{1} \mathbf{r} \\
\mathbf{D}_{2} \mathbf{r} \\
\mathbf{D}_{3} \mathbf{r} \\
\mathbf{D}_{4} \mathbf{r}
\end{array}\right)
$$

where, for all $i \in\{1, \ldots, 4\}, \mathbf{D}_{i}=\mathbf{U}_{i} \mathbf{F}_{i}$ where $\mathbf{F}_{i}$ represents the $i$-th prefiltering operation and $\mathbf{U}_{i}$ is the $i$-th $M$-band separable biorthogonal wavelet decomposition.

A robust reconstruction of $\mathbf{r}$ is then obtained as the solution of the minimization problem

$$
\inf _{\mathbf{r}} \sum_{i=1}^{4}\left\|\mathbf{c}_{i}-\mathbf{D}_{i} \mathbf{r}\right\|_{\mathbf{Q}_{i}}^{2}
$$

where $\mathbf{Q}_{i}$ is a positive self-adjoint operator and $\|\cdot\|_{\mathbf{Q}_{i}}^{2}=$ $\left\langle., \mathbf{Q}_{i}.\right\rangle$. The minimizer allows us to defines a generalized pseudo-inverse of the operator $\mathbf{D}_{\mathbf{c}}$ which is expressed as:

$$
\mathbf{D}_{\mathbf{c}}^{\sharp}=\left(\sum_{i=1}^{4} \mathbf{F}_{i}^{\dagger} \mathbf{U}_{i}^{\dagger} \mathbf{Q}_{i} \mathbf{U}_{i} \mathbf{F}_{i}\right)^{-1}\left(\mathbf{F}_{1}^{\dagger} \mathbf{U}_{1}^{\dagger} \mathbf{Q}_{1}, \ldots, \mathbf{F}_{4}^{\dagger} \mathbf{U}_{4}^{\dagger} \mathbf{Q}_{4}\right) \text {. }
$$

In practice, iterative approaches are generally necessary to compute $\mathbf{D}_{\mathbf{c}}{ }^{\sharp}$. Notice however that, by choosing $\mathbf{Q}_{i}=$ $\left(\mathbf{U}_{i} \mathbf{U}_{i}^{\dagger}\right)^{-1}$, the generalized pseudo-inverse takes a simple form, which can be easily implemented by combining the standard synthesis filter bank structures with filtering operations.

\section{EXPERIMENTAL RESULTS}

We apply these different decompositions to image denoising. More precisely, we aim at restoring the $512 \times 512$ Barbara image which is corrupted by an additive zero-mean white Gaussian noise. Four orthogonal decompositions are tested: the real dual-tree transform with dyadic (DTT2) and 4-band (DTT4) filter banks; the complex dual-tree transform with dyadic (DTT2cx) and 4-band (DTT4cx) filter banks. In this case, we use symlets of length 8 and 4-band filters of length 22. Moreover, we consider 4 additional biorthogonal transforms: the dual-tree transform with dyadic (DTTbi2) and 4band (DTTbi4) filter banks; the complex dual-tree transform with dyadic (DTTbi2cx) and 4-band (DTTbi4cx) filter banks. In this case, we use standard biorthogonal $5 / 3$ and $9 / 7$ wavelets and the associated 4-band basis functions corresponding to an equal-subband wavelet packet analysis. Classical estimators are applied to the resulting coefficients: Visushrink (Visu), SUREshrink (SURE) and the bivariate shrinkage (Biv)

\begin{tabular}{|c|c|c|c|c|}
\hline & Visu & SURE & Biv \\
\hline & DTT2 & 9.47 & 12.66 & 13.69 \\
\hline & DTT4 & 10.70 & 13.23 & 14.31 \\
\hline & DTT2cx & 9.72 & 12.84 & 13.95 \\
\hline & DTT4cx & 11.06 & 13.41 & 14.32 \\
\hline \multirow{4}{*}{$5 / 3$} & DTTbi2 & 8.55 & 11.65 & 12.73 \\
\hline & DTTbi4 & 9.39 & 12.14 & 13.45 \\
\hline & DTTbi2cx & 8.78 & 11.88 & 13.44 \\
\hline & DTTbi4cx & 9.71 & 12.35 & 13.82 \\
\hline \multirow{4}{*}{$9 / 7$} & DTTbi2 & 9.33 & 12.40 & 13.62 \\
\hline & DTTbi4 & 10.55 & 12.96 & 14.25 \\
\hline & DTTbi2cx & 9.56 & 12.59 & 13.97 \\
\hline & DTTbi4cx & 10.90 & 13.13 & 14.34 \\
\hline
\end{tabular}
[9]. Quantitative results are given in Table 1. Here, the initial SNR is equal to $5.67 \mathrm{~dB}$.

Table 1. Denoising results in terms of SNR (in $\mathrm{dB}$ ).

One can observe that, for all estimators, the 4-band structures always bring significant improvements (at least $0.2 \mathrm{~dB}$ ) with respect to the dyadic ones. Moreover, the complex decompositions outperform the real ones; the differences are however smaller with the bivariate shrinkage, for 4-band orthogonal filter banks. One can also see that the 9/7 biorthogonal decompositions provide results close to orthogonal ones. Nevertheless, biorthogonal decompositions could be more useful in image coding applications where they are known to be very effective.

\section{REFERENCES}

[1] I. W. Selesnick, R. G. Baraniuk, and N. G. Kingsbury, "The dual-tree complex wavelet transform," IEEE Signal Processing Magazine, pp. 123-151, Nov. 2005.

[2] E. Le Pennec and S. Mallat, "Sparse geometric image representations with bandelets," IEEE Trans. on Image Proc., vol. 14, no. 4, pp. 423-438, Apr. 2005.

[3] E. J. Candès and D. L. Donoho, Curves and Surfaces, chapter Curvelets - a surprisingly effective nonadaptive representation for objects with edges, pp. 105-120, Vanderbilt University Press, Nashville, TN, 1999.

[4] M. N. Do and M. Vetterli, "The contourlet transform: an efficient directional multiresolution image representation," IEEE Trans. on Image Proc., vol. 14, no. 12, pp. 2091-2106, Dec. 2005.

[5] N. G. Kingsbury, "The dual-tree complex wavelet transform: a new technique for shift invariance and directional filters," in Proc. IEEE Digital Signal Processing Workshop, Bryce Canyon, UT, USA, Aug. 9-12 1998, number 86.

[6] I. W. Selesnick, "Hilbert transform pairs of wavelet bases," Signal Processing Letters, vol. 8, no. 6, pp. 170-173, Jun. 2001.

[7] R. Yu and H. Ozkaramanli, "Hilbert transform pairs of biorthogonal wavelet bases," IEEE Trans. on Signal Proc., vol. 54, no. 6, pp. 2119-2125, June 2006.

[8] C. Chaux, L. Duval, and J.-C. Pesquet, "Image analysis using a dual-tree $M$-band wavelet transform," IEEE Trans. on Image Proc., vol. 15, no. 8, pp. 2397-2412, Aug. 2006.

[9] L. Şendur and I. W. Selesnick, "Bivariate shrinkage with local variance estimation," Signal Processing Letters, vol. 9, no. 12, pp. 438-441, Dec. 2002. 